INPLASY

PROTOCOL

To cite: Fan et al. Efficacy and safety of Xinkeshu in the treatment of angina pectoris of coronary heart disease: $A$ systematic review and metaanalysis protocol. Inplasy protocol 202190026. doi: 10.37766/inplasy2021.9.0026

Received: 08 September 2021

Published: 08 September 2021

Corresponding author: Maoxia Fan

fanmaoxia1127@163.com

Author Affiliation:

Affiliated Hospital of Shandong University of Traditional

Chinese Medicine

Support: Luke Zi [2018] No. 103.

Review Stage at time of this submission: The review has not yet started.

Conflicts of interest:

None declared.

\section{Efficacy and safety of Xinkeshu in the treatment of angina pectoris of coronary heart disease: A systematic review and meta-analysis protocol}

Fan, MX1;Tian, Y2; Guo, D³.

Review question / Objective: This study intends to provide a basis for systematically evaluating the efficacy and safety of Xinkeshu combined with conventional western medicine in the treatment of angina pectoris of coronary heart disease.

Condition being studied: A systematic review of the effectiveness and safety of Xinkeshu in treating coronary heart disease and angina pectoris.

Information sources: CNKI, Wanfang, VIP, Web of Science, PubMed, Cochrane Library and EMbase databases were searched for the period from the establishment of the database to August 31,2021 . References that meet the inclusion criteria of the study intended to be reviewed one by one to avoid omissions. XKS and AP of CHD and their synonyms were set as search words. Contact with authors: Maoxia Fan, Ying Tian and Dong Guo.

INPLASY registration number: This protocol was registered with the International Platform of Registered Systematic Review and Meta-Analysis Protocols (INPLASY) on 08 September 2021 and was last updated on 08 September 2021 (registration number INPLASY202190026).

\section{INTRODUCTION}

Review question / Objective: This study intends to provide a basis for systematically evaluating the efficacy and safety of Xinkeshu combined with conventional western medicine in the treatment of angina pectoris of coronary heart disease.

Condition being studied: A systematic review of the effectiveness and safety of 
Xinkeshu in treating coronary heart disease and angina pectoris.

\section{METHODS}

Participant or population: Patients with coronary heart disease and angina pectoris.

Intervention: Xinkeshu capsules or Xinkeshu tablets or Xinkeshu decoction combined with conventional western medicine.

Comparator: Conventional western medicine: the main drugs are anticoagulation, antiplatelet aggregation, $\beta$-receptor blockers, nitrates, angiotensinconverting enzyme inhibitors (ACEI), statins and other drugs for blood lipid regulation.

Study designs to be included: Randomized controlled trial.

Eligibility criteria: 1.1 Type RCT of AP of CHD treated by XKS combined with conventional western medicine.1.2 Objects The diagnostic criteria of AP of CHD can be met according to the diagnostic criteria of syndrome elements of AP of CHD. In addition, there are no restrictions on age, sex, history of tobacco and alcohol and so on.1.3 Intervention measures The control group was only treated with the same routine western medicine. In clinical, the main drugs included anticoagulation, antiplatelet aggregation, $\beta$-receptor blockers, nitrates, angiotensin converting enzyme inhibitor (ACEI), statins regulating blood lipids and other drugs. At the same time, the treatment group was treated with XKS capsule or XKS tablet or XKS decoction combined with conventional western medicine.1.4 Outcome index The criteria for judging the expected outcome were clear, including the frequency of angina pectoris, the duration of angina pectoris, the dosage of nitroglycerin and the curative effect of ECG (total effective rate = markedly effective + effective). The secondary indicators were the efficacy of angina pectoris (total effective rate $=$ significant + effective), blood lipids (triglyceride, total cholesterol, low density lipoprotein cholesterol, high density lipoprotein cholesterol), hemorheology (whole blood viscosity, plasma viscosity, fibrinogen), serum factors (C-reactive protein, endothelin-1, homocysteine, nitric oxide), adverse drug reactions.

Information sources: CNKI, Wanfang, VIP, Web of Science, PubMed, Cochrane Library and EMbase databases were searched for the period from the establishment of the database to August 31, 2021. References that meet the inclusion criteria of the study intended to be reviewed one by one to avoid omissions. XKS and AP of CHD and their synonyms were set as search words. Contact with authors: Maoxia Fan, Ying Tian and Dong Guo.

Main outcome(s): Frequency of angina pectoris;Duration of angina attack;ECG efficacy;Blood lipids (triacylglycerol, total cholesterol, low density lipoprotein cholesterol, high density lipoprotein cholesterol);Serum factors (the C-reactive protein, endothelin-1, homocysteine, nitric oxide), adverse events or adverse drug reactions.

Quality assessment / Risk of bias analysis: The risk of bias in the included literature was evaluated according to Cochrane Reviewer Manual 5.01. Evaluation content includes: random sequence generation, allocation plan hiding, blind method, data integrity and selective report results. The literature quality evaluation is carried out by two evaluators independently according to a unified quality standard. If there is any disagreement, the two parties will discuss and resolve or consult the third evaluator.

Strategy of data synthesis: The Revman5.3 software provided by the Cochrane website was used for analysis. Categorical variables are expressed by odds ratio (OR) and marked with $95 \%$ confidence interval (CI). Continuous variables are expressed by mean difference (MD) and marked with $95 \% \mathrm{Cl}$. If $\mathrm{P0.1}$ or $\mathrm{I}^{2}<50 \%$, it means that the heterogeneity between groups is small, and the fixed effects model is used for combined analysis; when P50\%, It shows that the heterogeneity between the groups 
is large, and the random effects model is used for combined analysis, and the results are shown in forest plots. Analysis of potential publication bias is shown in a funnel chart, and sensitivity analysis and subgroup analysis are used when necessary.

Subgroup analysis: A subgroup analysis would be conducted according to different interventions, participants, gender, treatment duration and drug dose, to explore the source of heterogeneity if there were at least 10 trials included.

Sensitivity analysis: Sensitivity analysis would be conducted according to sample size, missing data and methodological quality to identify the quality.

Country(ies) involved: China.

Keywords: Xinkeshu; angina pectoris of coronary heart disease; systematic review; protocol.

Contributions of each author:

Author 1 - Maoxia Fan.

Email: fanmaoxia1127@163.com

Author 2 - Ying Tian.

Email: tianying2021666@163.com

Author 3 - Dong Guo.

Email: guodong2021666@163.com 